www.cahiersagricultures.fr

\title{
Remerciements aux relecteurs 2016
}

La revue Cahiers Agricultures remercie tous les relecteurs qui ont bien voulu l'aider à prendre une décision sur la publication des textes et ont fourni aux auteurs de précieux conseils.

\author{
Gilles Allaire \\ Hichem Amichi \\ Véronique Ancey \\ Valérie Angeon \\ Xavier Arnauld de Sartre \\ Xavier Augusseau \\ Abdon Awono \\ Gérard Azoulay \\ Mohamed Nasser Baco \\ Sébastien Bainville \\ Jean-Marc Barbier \\ Mamadou Billo Barry \\ Marouane Baslam \\ Didier Bazile \\ Agnès Bégué \\ Jean-François Bélières \\ Salah Eddine Benziouche \\ Rachel Bezner Kerr \\ Anne Bichard \\ Chris Blackmore \\ Yahia Bouabdellaoui \\ Boualem Brahim Bouammar \\ Bassou Bouazzama \\ Christian Bouquet \\ Alain Bourbouze \\ Zhour Bouzidi \\ Florence Brondeau \\ Jan Brouwers \\ Gilles Brunschwig \\ Perrine Burnod \\ Luc Cambrezy \\ François Casabianca \\ Pascal Chevalier \\ Bruno Cheviron \\ Eduardo Chia \\ Salah Chouaki \\ Daniele Clavel \\ François Clinard \\ Monica Commandeur \\ Christian Corniaux \\ Yacouba Coulibaly \\ Teresa Crespo \\ Benoît Dedieu \\ Chantal Delooz \\ Marco d'Errico \\ Jean-Louis Devineau \\ Marijke d'Haese \\ Mohamed Koulam Dicko
}

François Doligez

Elodie Dorey

Bruno Dorin

Isabelle Droy

Patrick Dugué

Michel Dulcire

Fernando Eguren

Corinne Eychenne

Baalam Facho

Guy Faure

Bernard Faye

Laurene Feintrenie

Fabien Ferchaud

Anne Floquet

Stéphane Fournier

Remi Gaudin

Denis Gautier

Françoise Gérard

David Gibbon

Martine Guibert

Andy Hall

Mariusz Hamulczuk

Tarik Hartani

Michel Havard

Alain Havet

Bernard Hubert

Johann Huguenin

Christian Huyghe

Jean-Yves Jamin

Pierre Janin

Philippe Jeanneaux

Janice Jiggins

Damien Jourdain

Philippe Jouve

Ahcene Ahcène Kaci

Hamadé Kagoné

Conchita M. G Kêdowidé Mêvo Guézo

Andrea Knierim

Cokou Patrice Kpadé

Marcel Kuper

Julie Labatut

Guillaume Lacquement

Frédéric Lançon

Etienne Landais

Frédéric Landy

Jérôme Lazard

Fabrice Le Bellec

Pierre-Yves Le Gal
Philippe Lebailly

Kaouthar Lebdi-Grissa

Guillaume Leturcq

Patrice Levang

Toufik Madani

Géraud Magrin

Pascale Maizi

Stefan Mann

Anaïs Marshall

Thibaud Martin

Dominique Masse

Anne Mathieu

Pierre-Louis Mayaux

Armelle Mazé

Doyle McKey

Benoit Mégevand

Ismail Moumouni

François Moutou

Suzanne Nederlof

Aimé Joseph Nianogo

François Papy

Jean-Louis Pham

Florence Phocas

Didier Picard

Jean-Pascal Pichot

René Poccard-Chapuis

Giovanni Prete

Nicolas Puig

Hassan Quarouch

Alain Ratnadass

Christine Rawski

Brigitte Remy

Camille Renaudin

Daniel Ricard

Xavier Richet

Laurent Rieutort

Alain Rival

Niels Röling

Averill Livia Roy

François Ruf

Philippe Ryckewaert

Julie Ryschawy

Eric Sabourin

Gabriel Sangli

Joel Scher

Georges Serpantie

Pierre Silvie

Nkrintra Singhrattna 
Cah. Agric. 2016, 25, 610001

Jean-Michel Sourisseau Mohamed Taher Srairi Cecilia Tacoli

Abdelilah Taky Bernard Claude Tallet Leopold Tatsadjieu Fabrice Teletchea
Aurélie Toillier

Jean Philippe Tonneau

Edmond Totin

Jean-François Tourrand

Bernard Toutain

Isabelle Vagneron

Eric Vall
Gilles Sylvanus Vias

Pierre Vissoh

Marion Vittecoq

Betty Wampfler

Elisabeth Pawindé Zida

Jean Sibiri Zoundi 